\title{
Signal Detection for Molecular MIMO Communications with Asymmetrical Topology
}

\author{
Cong Wu, Lin Lin, Weisi Guo, and Hao Yan
}

\begin{abstract}
Molecular communication (MC) has attracted people's attention due to its potential applications at the micro- to nano-scale. In MC, the transmission rate is usually very low due to the slow diffusion of information molecules and therefore multiple-input multiple-output (MIMO) system is introduced. However, severe interference occurs when the same types of information molecules are used at different transmission antennas. Up to now, most literature focuses on MIMO systems with symmetrical topology. In this paper, a molecular MIMO communication system with asymmetrical topology, where the number of transmission antennas is not equal to that of the reception antennas, is investigated. The zero-forcing $(\mathrm{ZF})$ detection approach is proposed and discussed for three cases, i.e., the number of transmission antennas is smaller than, equal to and larger than the number of the reception antennas. Considering the inter-link interference (ILI) and the inter-symbol interference (ISI), the error probability of ZF detection is derived and comparisons are made with existing molecular MIMO detection method. Besides, the adaptive observation time for each reception antenna is derived for better performance. Numerical results show that ZF detection performs better than the existing molecular MIMO detection method when the ILI is large.
\end{abstract}

Index Terms-molecular communication, MIMO, asymmetric topology, signal detection, error performance.

\section{INTRODUCTION}

$\mathbf{M}$ OLECULAR communication (MC) is a promising paradigm which is considered to be an alternative communication method for nanomachines, especially for the complicated nanonetworks [1]-[3]. Recently, it attracts great interests of researchers due to the biocompatibility and low energy consumption compared with traditional electromagnetic wireless communication [4]. There are many potential applications by using MC, such as drug delivery systems in the human body [5], [6], environmental preservation [7], artificial immune system [8] and cell-cell communication [9]. Usually, in diffusive MC, molecules are the information carrier, which spread slowly in the liquid or gaseous media. The slow diffusion significantly restricts the transmission rate [10] and

This work was supported in part by National Natural Science Foundation, China (61971314), in part by Natural Science Foundation of Shanghai (19ZR1426500), in part by Science and Technology Commission of Shanghai Municipality (19510744900), and in part by the EU Horizon 2020 Programme (792799). (Corresponding author: Lin Lin)

C. Wu and L. Lin are with the College of Electronics and Information Engineering, Tongji University, Shanghai 201804, China (e-mail: 1832911@tongji.edu.cn; fxlinlin@tongji.edu.cn).

W. Guo is with Cranfield University, MK43 0AL, U.K. (e-mail: weisi.guo@cranfield.ac.uk).

H. Yan is with the School of Electronic, Information and Electrical Engineering, Shanghai Jiao Tong University, Shanghai 200240, China (e-mail: yan_hao@sjtu.edu.cn).

L. Lin is also with the University of Warwick, CV4 7AL, U.K. multiple-input multiple-output (MIMO) systems are designed to solve this problem.

In the MC literature, the problem of MIMO communication has been widely discussed. MIMO technology was first introduced to MC in [11]. Specifically, transmit diversity and three receive diversity combining strategies were proposed. Moreover, spatial multiplexing with particular transmission pairs (e.g., reception antenna 1 receives the signal sent by transmission antenna 1 , reception antenna 2 receives the signal sent by transmission antenna 2, etc.) over a $M \times M$ MIMO system was considered and a better performance on throughput can be obtained. Spatial modulation techniques for molecular MIMO communication were further investigated in [12] and [13], and were expected to achieve better error performance when given the same symbol rate. In [14], novel index-based schemes are provided and are expected to combat inter-symbol interference (ISI) and interlink interference (ILI) efficiently. As for channel analysis, the authors in [15] proposed two channel estimations methods for $M \times M$ molecular MIMO communication, i.e., maximum likelihood and least-squares estimation.

Unlike the $M \times M$ systems, a $2 \times 2$ molecular MIMO communication system with absorbing receiver antenna was considered in [16]-[18]. In [16] and [17], channel parameters were fitted according to the simulation data since hitting the two different reception spheres becomes dependent events. Four detection algorithms were introduced and the testbed for the considered MIMO system was shown. In [18], Alamoutitype coding and repetition MIMO coding were applied at the transmission side. At the receiver-side, selection diversity and equal-gain combination were used as combining strategies. For the channel impulse response (CIR), a trained artificial neural network was utilized to get the mean channel coefficients. Combined with the molecular motor communication channel, the authors in [19] investigated the performance and the throughput of a $2 \times 2$ MIMO system.

Besides, machine learning methods were introduced to molecular MIMO communication system to solve some problems that are difficult to obtain theoretical solutions [20]-[22]. In [20] and [21], to expand the channel model from singleinput single-output (SISO) to MIMO, artificial neural network (ANN) was trained to estimate the channel parameters after fitting the channel model parameters. In [22], an exact analytical framework for the MC system that relies on the perfect knowledge of the channel model was developed. By using ANN, receivers have similar performance to the theoretical derivation with the exact channel parameters, in terms of error probability. 
Most of those literature focuses on the system model with symmetrical topology, i.e., the number of the transmission antennas and the number of the reception antennas are equal. However, MIMO communication system with asymmetrical topology is an interesting scenario which makes the system design more flexible and has been widely discussed in traditional wireless communications. Besides, even if the standard of molecular MIMO communication is established, the molecular transceiver products from the same company or different companies may have different numbers of antennas. It is meaningful and efficient for these molecular transceivers with asymmetrical topology (with different number of antennas) to communicate with each other, no matter they are in the same communication network or heterogeneous networks. Moreover, when applying those detection methods for molecular MIMO communication system with symmetrical topology, information resources will not be fully utilized since some antennas are not used.

In this paper, signal detection and error performance of a molecular MIMO communication system with asymmetrical structure are investigated. We propose the zero-forcing (ZF) detection approach for the considered system with asymmetrical topology. In contrast to [11] and [17], we focus on communication systems with asymmetrical topology. And the signals from all of the reception antennas are used to demodulate transmitted signals, which means there are no certain pairs in our consideration. The major contributions in this paper include:

- The ZF detection approach, where detection is made based on the results after matrix processing, is introduced to eliminate ILI in the considered molecular MIMO communication system with asymmetrical topology. The mean and variance of ISI are derived.

- The peak concentration time of the superposition of CIRs for each reception antenna is derived and selected as an adaptive observation time. Both the fixed observation time and adaptive observation time are combined with the detection schemes to evaluate the performance.

- Considering both ILI and ISI, error performance of ZF detection is investigated and compared with existing molecular MIMO methods.

The rest of this paper is organized as follows. In Section II, the system model is introduced. Section III presents the ZF detection approach and the performance analysis without ISI is given in Section IV. In Section V, the mean and variance of ISI are provided. ILI as well as ISI are considered to evaluate the entire performance. Numerical results are provided in Section VI. Finally, Section VII concludes the paper.

\section{System ModeL}

A molecular MIMO communication system with asymmetrical topology is shown in Fig. 1, where the transmitter employs $M$ transmission antennas and the receiver employs $N$ reception antennas. The transmitter and the receiver are assumed to be well synchronized [23], [24]. It is assumed that all the transmission antennas and reception antennas distribute on two parallel straight lines. The distance between

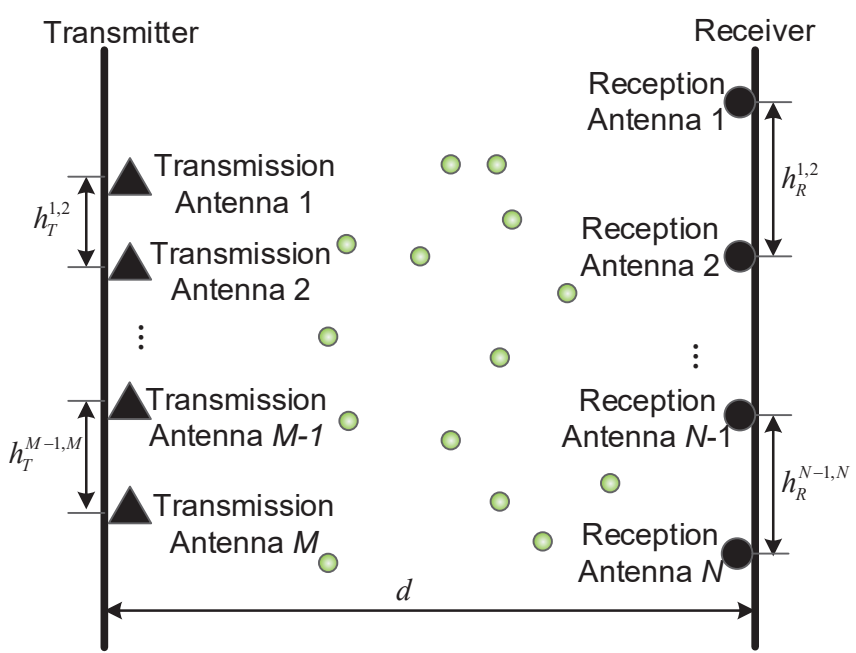

Fig. 1. System model of the molecular MIMO communication with $M$ transmission antennas and $N$ reception antennas. Information molecules are denoted by green spheres.

the transmitter and the receiver is $d$ and the distance between the adjacent transmission antennas (antenna $i-1$ and antenna $i$ ) is denoted as $h_{T}^{i-1, i}$. Similarly, the distance between the adjacent reception antennas (antenna $j-1$ and antenna $j$ ) is denoted as $h_{R}^{j-1, j}$.

At the transmitter side, the transmitted information is split into $M$ streams that are then fed to the corresponding $M$ transmission antennas. Here we assume that the information bits are uniformly allocated to the transmission antennas in sequence. Fig. 2 illustrates the process of turning a transmitted bit sequence into bit streams. For the transmission of each bit stream at an individual transmission antenna, On-Off Keying (OOK) modulation is employed, which means $Q$ molecules are released for sending "1" while no molecules are released for sending " 0 ". At the beginning of each symbol interval, the $M$ transmission antennas release $Q$ or 0 molecules simultaneously according to the bits they send. Once emitted, the information molecules move in the media through free diffusion with diffusion coefficient $D$, which can be described by Fick's second law. In the biological world, oxygen, carbon dioxide and benzene molecules can pass through the cell membrane by passive transport [25, Chapter 2]. Moreover, in [26], planar laser induced fluorescence and high-speed cameras are used to obtain the information strength at the reception side without influencing the propagation of molecules. So in this paper, we assume that all the reception antennas have spherical shapes with radius $r$ and are considered as "passive" antennas which can count the number of the molecules without influencing the motion of them [27]-[30]. Thus, the CIR, i.e., the probability of observing molecule in the $j$ th reception antenna of the receiver in response to one molecule's input from the $i$ th transmission antenna can be given by [28]

$$
h_{i j}(t)=\frac{V_{R}}{(4 \pi D t)^{\frac{3}{2}}} \exp \left(-\frac{d_{i j}^{2}}{4 D t}\right),
$$

where $d_{i j}$ is the distance between the $i$ th transmission antenna and the $j$ th reception antenna, $V_{R}$ is the volume of the 


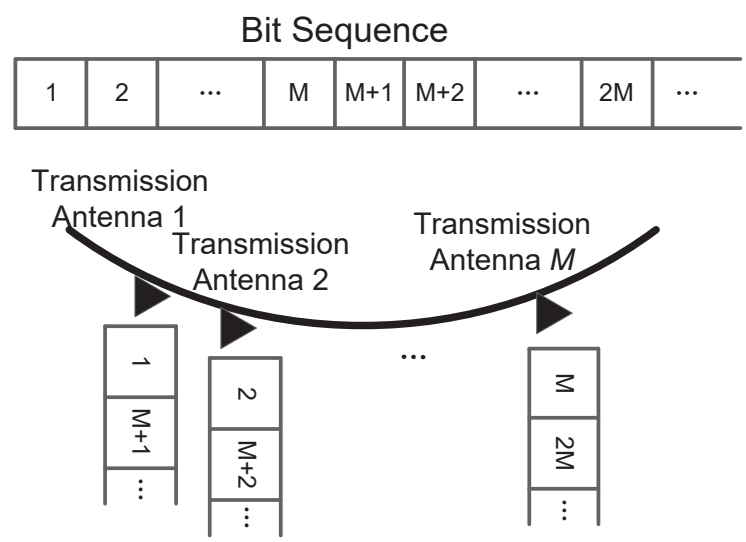

Fig. 2. Diagram of the generation of bit streams. The numbers in the square represent the orders of the bits.

reception antenna.

It should be noted that each molecular emission at the transmission antenna can cause responses at all of the reception antennas. Therefore, in the $k$ th symbol, the received molecular number at the $j$ th reception antenna $r_{j}\left(k, t_{s}\right)$, abbreviated as $r_{j}(k)$, at time $t_{s}$ after the beginning of the symbol in response of the transmitted symbols from all transmission antennas, can be written as

$$
r_{j}(k) \triangleq r_{j}\left(k, t_{s}\right)=\sum_{i=1}^{M} s_{i}(k) h_{i j}\left(t_{s}\right)+n_{j}(k),
$$

where $s_{i}(k)$ is the transmitted symbol from $i$ th transmission antenna and equals to $Q$ or $0, n_{j}(k)$ is the corresponding noise at the reception antenna. Noise molecules could come from all transmitters not considered in model or some other external sources. Its number can be modelled as a Poisson random variable with mean $\mu_{n}$ [31], which can be approximated as an Gaussian random variable when the number of molecules is large enough, e.g. larger than 100. In addition, to avoid severe interference between two MC systems, or between the considered $\mathrm{MC}$ system and the external noise sources, they will be separated for a long distance when the same information molecules are used. Therefore we assume the distance between the external noise sources and the considered communication system is large enough and thus the noises at the $N$ reception antennas are relatively stationary and have the same mean. In this paper, $n_{j}(k)$ can be expressed as [32]

$$
n_{j}(k) \sim \mathcal{N}\left(\mu_{n}, \sigma_{n}^{2}\right)
$$

where $\sigma_{n}^{2}=\mu_{n}$ is the variance of the noise and $\mathcal{N}$ denotes the normal distribution.

The length of the transmitted bit sequence is assumed to be $q M$, and $M$ parallel transmissions are exploited, each transmitting $q$ bits. Meanwhile, the long tail of the CIR in (1) brings a non-neglectable influence in the following time slots. In other words, the ISI occurs. Then we write all the transmissions in the channel in matrix format as

$$
\mathbf{R}=\mathbf{H S}+\mathbf{I S I}+\mathbf{N}
$$

where $\mathbf{R}, \mathbf{H}, \mathbf{S}, \mathbf{N}$ and ISI are the received signal matrix, the channel matrix, the transmitted signal matrix, the noise matrix and the ISI matrix, respectively. They can be expressed as

$$
\begin{aligned}
& \mathbf{R}=\left[\begin{array}{cccc}
r_{1}(1) & r_{1}(2) & \cdots & r_{1}(q) \\
r_{2}(1) & r_{2}(2) & \cdots & r_{2}(q) \\
\vdots & \vdots & \ddots & \vdots \\
r_{N}(1) & r_{N}(2) & \cdots & r_{N}(q)
\end{array}\right] \\
& \mathbf{H}=\left[\begin{array}{cccc}
h_{11}\left(t_{s}\right) & h_{21}\left(t_{s}\right) & \cdots & h_{M 1}\left(t_{s}\right) \\
h_{12}\left(t_{s}\right) & h_{22}\left(t_{s}\right) & \cdots & h_{M 2}\left(t_{s}\right) \\
\vdots & \vdots & \ddots & \vdots \\
h_{1 N}\left(t_{s}\right) & h_{2 N}\left(t_{s}\right) & \cdots & h_{M N}\left(t_{s}\right)
\end{array}\right], \\
& \mathbf{S}=\left[\begin{array}{cccc}
s_{1}(1) & s_{1}(2) & \cdots & s_{1}(q) \\
s_{2}(1) & s_{2}(2) & \cdots & s_{2}(q) \\
\vdots & \vdots & \ddots & \vdots \\
s_{M}(1) & s_{M}(2) & \cdots & s_{M}(q)
\end{array}\right] \\
& \mathbf{N}=\left[\begin{array}{cccc}
n_{1}(1) & n_{1}(2) & \cdots & n_{1}(q) \\
n_{2}(1) & n_{2}(2) & \cdots & n_{2}(q) \\
\vdots & \vdots & \ddots & \vdots \\
n_{N}(1) & n_{N}(2) & \cdots & n_{N}(q)
\end{array}\right] \\
& \mathbf{I S I}=\left[\begin{array}{cccc}
I S I_{1}(1) & I S I_{1}(2) & \cdots & I S I_{1}(q) \\
I S I_{2}(1) & I S I_{2}(2) & \cdots & I S I_{2}(q) \\
\vdots & \vdots & \ddots & \vdots \\
I S I_{N}(1) & I S I_{N}(2) & \cdots & I S I_{N}(q)
\end{array}\right]
\end{aligned}
$$

The ISI component at reception antenna $j$ in the $z$ th time slot can be expressed as

$$
I S I_{j}(z)=\sum_{k=1}^{z-1} \sum_{i=1}^{M} s_{i}(k) h_{i j}\left(t_{s}+k T\right)
$$

where $s_{i}(k)=$ " 0 " or " $Q$ " is the element in matrix $\mathbf{S}$ and denotes the number of molecules emitted in the $k$ th symbol interval at transmission antenna $i, T$ is the symbol duration.

It can be seen from (2) that the signal at the reception antenna contains the summation of all $M$ components from the transmission antennas. Thus, ILI exists. In the next section, a signal detection scheme is proposed to eliminate the ILI and realize detection with low error probability.

\section{Proposed Signal Detection Scheme}

In this section, the $\mathrm{ZF}$ detection approach for molecular MIMO system with asymmetrical topology is proposed. We focus on the elimination of the influence of the ILI by matrix operations in ZF detection and temporarily ignore ISI in (10) by supposing a large symbol duration $T$. The consideration of both ILI and ISI will be investigated in Section V. In this section, three cases, i.e., $M<N, M=N$ and $M>N$ are discussed. 


\section{A. Case 1: $M<N$}

In this subsection, the case where the number of transmission antennas is smaller than the reception antennas is discussed.

Note that each row in channel matrix $\mathbf{H}$ represents the CIRs for one certain reception antenna. Meanwhile, different positions of the transmission antennas ensures that there will not be two same columns or two columns have linear relationship in matrix $\mathbf{H}$. In other words, $\mathbf{H}$ has independent columns. So matrix $\mathbf{H}$ has row full rank which is

$$
\operatorname{rank}(\mathbf{H})=M \text {. }
$$

The ILI is reduced by premultiplying the received signal matrix $\mathbf{R}$ by the Moore-Penrose pseudo inverse [33] of the channel matrix $\mathbf{H}$ as

$$
\widetilde{\mathbf{R}}=\mathbf{H}^{+} \mathbf{R},
$$

where $\mathbf{H}^{+}$denotes the Moore-Penrose pseudo inverse of the channel matrix and is defined as

$$
\mathbf{H}^{+} \triangleq\left(\mathbf{H}^{\mathbf{H}} \mathbf{H}\right)^{-1} \mathbf{H}^{\mathbf{H}}
$$

when the matrix has linear independent columns, and $\mathbf{H}^{\mathbf{H}}$ denotes the Hermitian transpose of $\mathbf{H}$. Since each element in the channel matrix in (6) has no complex component, i.e., $\mathbf{H}$ is a real matrix, the Hermitian transpose is equivalent to the transpose of one matrix [34]. So we rewrite (13) as

$$
\mathbf{H}^{+} \triangleq\left(\mathbf{H}^{\mathbf{T}} \mathbf{H}\right)^{-1} \mathbf{H}^{\mathbf{T}}
$$

where $\mathbf{H}^{\mathbf{T}}$ denotes the transpose of $\mathbf{H}$.

The effectiveness of this approach in reducing ILI can be seen by substituting (14) into (12), which results in

$$
\begin{aligned}
\widetilde{\mathbf{R}} & =\left(\mathbf{H}^{\mathbf{T}} \mathbf{H}\right)^{-1} \mathbf{H}^{\mathbf{T}} \mathbf{R} \\
& =\left(\mathbf{H}^{\mathbf{T}} \mathbf{H}\right)^{-1} \mathbf{H}^{\mathbf{T}}(\mathbf{H S}+\mathbf{N}) \\
& =\left(\mathbf{H}^{\mathbf{T}} \mathbf{H}\right)^{-1}\left(\mathbf{H}^{\mathbf{T}} \mathbf{H}\right) \mathbf{S}+\left(\mathbf{H}^{\mathbf{T}} \mathbf{H}\right)^{-1} \mathbf{H}^{\mathbf{T}} \mathbf{N} \\
& =\mathbf{S}+\left(\mathbf{H}^{\mathbf{T}} \mathbf{H}\right)^{-1} \mathbf{H}^{\mathbf{T}} \mathbf{N} \\
& =\mathbf{S}+\widetilde{\mathbf{N}},
\end{aligned}
$$

where matrix $\widetilde{\mathbf{R}}$ is composed of the initial signal component $\mathbf{S}$ without ILI and the transformed noise matrix $\widetilde{\mathbf{N}}$. $\widetilde{\mathbf{R}}$, as well as $\widetilde{\mathbf{N}}$, are both $M \times q$ matrices, with the same dimension as $\mathbf{S}$, but different from $\mathbf{R}$. The $k$ th column in matrix $\widetilde{\mathbf{R}}$ represents the signal before making a decision in the $k$ th symbol interval, which can be expressed as

$$
\widetilde{\mathbf{r}}(k)=\mathbf{s}(k)+\widetilde{\mathbf{n}}(k),
$$

where $\widetilde{\mathbf{s}}(k)$ and $\widetilde{\mathbf{n}}(k)$ are the $k$ th column in $\mathbf{S}$ and $\widetilde{\mathbf{N}}$, respectively. Also note that both $\widetilde{\mathbf{r}}(k)$ and $\widetilde{\mathbf{n}}(k)$ are $M \times 1$ vectors where $M$ is the number of transmission antennas. If we pay attention to the detection for one certain symbol at the $i$ th transmission antenna, i.e., the $i$ th element in $\widetilde{\mathbf{r}}(k)$, which denoted by $\widetilde{r}_{i}(k)$, it can be expressed as

$$
\widetilde{r}_{i}(k)=s_{i}(k)+\tilde{n}_{i}(k), \quad i \in\{1, \ldots, M\},
$$

where $\widetilde{n}_{i}(k)$ are the $i$ th element in $\widetilde{\mathbf{n}}(k)$.

Theorem 1: The $i$ th element in the transformed noise vector, denoted by $\tilde{n}_{i}(k)$, has a Gaussian distribution.
Proof 1: The transformed noise matrix $\widetilde{\mathbf{N}}$ in (15) is generated by premultiplying the noise matrix $\mathbf{N}$ by $\mathbf{H}^{+}$, a $M \times N$ matrix. If we denote the element in the $i$ th row and $j$ th column of the pseudo inverse matrix $\mathbf{H}^{+}$as $h_{i j}^{\prime}$, then $\widetilde{n}_{i}(k)$ can be expressed as the summation of the $k$ th column of $\mathbf{N}$ in (8) with weight from the $i$ th row of $\mathbf{H}^{+}$, which can be expressed as

$$
\widetilde{n}_{i}(k)=\sum_{j=1}^{N} h_{i j}^{\prime} n_{j}(k),
$$

where $n_{j}(k)$ has a Gaussian distribution as mentioned in (3). Once the topology of the molecular MIMO system and observation time are determined, the CIRs are determined for attribute $i$ and $j$, and therefore the channel matrix $\mathbf{H}$ is determined. Thus, each element in $\mathbf{H}^{+}$is determined. Besides, based on the independent Brownian movement of molecules in the medium, the noise is independent at each reception antenna [32]. So $\widetilde{n}_{i}(k)$ can be seen as a weighted summation of $N$ independent Gaussian variables and therefore follows a Gaussian distribution. The mean and variance of $\widetilde{n}_{i}(k)$ can be expressed as

$$
\begin{aligned}
& E\left(\widetilde{n}_{i}(k)\right)=\sum_{j=1}^{N} h_{i j}^{\prime} \mu_{n}, \\
& D\left(\widetilde{n}_{i}(k)\right)=\sum_{j=1}^{N} h_{i j}^{\prime 2} \sigma_{n}^{2} .
\end{aligned}
$$

It can be seen from (20) that the $\mathrm{ZF}$ method enhances the noise. But on the other hand, the enhancement is finite and depends on the elements in $\mathbf{H}^{+}$, which are directly related to the CIRs in $\mathbf{H}$. Thus, when channel parameters are determined, stronger signal strength (the number of molecules when transmitting " 1 ") can effectively combat with the noise enhancement to achieve a better error performance.

As shown in (17), the detected signal $\widetilde{r}_{i}(k)$ fluctuates over the transmitted signal $s_{i}(k)$ due to the influence of $\widetilde{n}_{i}(k)$. And since $s_{i}(k)$ may have two values, i.e., 0 and $Q$, which are corresponding to the transmitted signal, the detection process turns into figuring out the value of $s_{i}(k)$ under the interference of a Gaussian noise, which makes the detection easily achieved by a simple threshold detector.

\section{B. Case 2: $M=N$}

In this subsection, the case when the number of transmission antennas and the reception antennas are equal, is investigated. The channel matrix $\mathbf{H}$ then turns into a square matrix and has full rank as

$$
\operatorname{rank}(\mathbf{H})=M=N .
$$

So the Moore-Penrose pseudo inverse of the channel matrix turns to be the inverse of it. The $\mathrm{ZF}$ detection in this case can be seen as a special case of Case 1 and the ILI can be eliminated similarly.

Meanwhile, the detection method in [11] where pairs are assumed to transmitting and receiving signals, can also be applied in this case, which we called detection with transmission pairs in the following. The error probability of this method will be introduced in Section IV. 


\section{Case 3: $M>N$}

In this subsection, we consider the case where the number of transmission antennas is larger than that of the reception antennas. Obviously, the transmission antennas and the reception antennas cannot constitute pairs in this case. So we discuss the $\mathrm{ZF}$ detection in this case.

In this case, although the channel matrix is still a $N \times M$ matrix, it has row full rank (column full rank in Case 1 ) since $M>N$ as

$$
\operatorname{rank}(\mathbf{H})=N
$$

Its pseudo inverse becomes

$$
\mathbf{H}^{+} \triangleq \mathbf{H}^{\mathbf{H}}\left(\mathbf{H}^{\mathbf{H}} \mathbf{H}\right)^{-1} \text {. }
$$

Similarly, since no complex component exists in (23), the Hermitian transpose is equivalent to the transpose of it. So we rewrite it as

$$
\mathbf{H}^{+} \triangleq \mathbf{H}^{\mathbf{T}}\left(\mathbf{H}^{\mathbf{T}} \mathbf{H}\right)^{-1} .
$$

So if we apply the ZF detection in this case, i.e, premultiplying the received signal matrix by the Moore-Penrose pseudo inverse of the channel matrix $\mathbf{H}$ as

$$
\begin{aligned}
\widetilde{\mathbf{R}} & =\mathbf{H}^{+} \mathbf{R} \\
& =\mathbf{H}^{\mathbf{T}}\left(\mathbf{H}^{\mathbf{T}} \mathbf{H}\right)^{-1} \mathbf{R} \\
& =\mathbf{H}^{\mathbf{T}}\left(\mathbf{H}^{\mathbf{T}} \mathbf{H}\right)^{-1} \mathbf{H S}+\mathbf{H}^{\mathbf{T}}\left(\mathbf{H}^{\mathbf{T}} \mathbf{H}\right)^{-1} \mathbf{N} .
\end{aligned}
$$

We can no longer obtain the initial signal matrix component, i.e., the ZF detection cannot be used to obtain the original transmitted signal matrix for the case $M>N$.

Intuitively, the number of transmission bit streams is larger than the reception streams, which indicates $M$ variables $(M$ transmitted bit symbols) need to be solved by $N$ equations (equations between the input signals and output signals) and the unique solution cannot be obtained. In other words, the signal at the receiver cannot be demodulated successfully using $\mathrm{ZF}$ detection. However, if high transmission rate is not pursued, this case can be widely used in spatial diversity scenario, where multiple copies of one data stream are transmitted at a number of antennas and various received responses of the data are exploited to improve the reliability of transmission. Spacetime block codes, such as Alamouti codes [35] and linear dispersion codes [36] are widely used. Alamouti codes have been introduced to MC in [18] and some other diversity techniques still worth investigation.

\section{Decision Rules and Adaptive Sampling Time}

The expectations when transmitting " 0 " and " 1 " are denoted by $\mu_{0}$ and $\mu_{1}$, respectively. They have the following expressions

$$
\begin{gathered}
\mu_{0}=E\left(\widetilde{n}_{i}(k)\right), \\
\mu_{1}=Q+E\left(\widetilde{n}_{i}(k)\right) .
\end{gathered}
$$

The threshold $\xi^{*}$ can be calculated by [37, Fig. 3]

$$
\xi^{*}=\arg \min _{\xi}[P(1 \mid 0)+P(0 \mid 1)], \quad \xi \in[0,+\infty],
$$

where $\xi$ are all the possible threshold we can choose, $P(1 \mid 0)$ and $P(0 \mid 1)$ are the error probabilities when transmitting " 0 " and " 1 ", respectively.

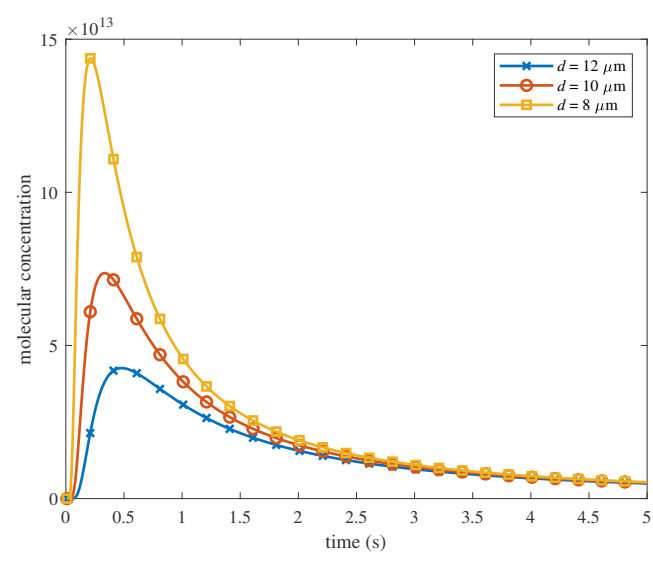

(a) Different CIRs.

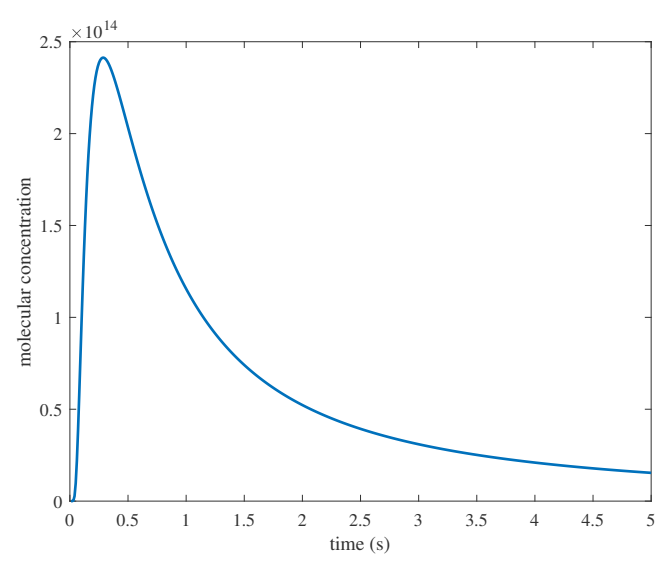

(b) Superposition of the CIRs.

Fig. 3. Different CIR curves and the superposition of them. The diffusion coefficient $D$ is $50 \mu \mathrm{m}^{2} / \mathrm{s}$.

Thus, the corresponding decision rule can be written as

$$
s_{i}(k)= \begin{cases}0 & \widetilde{r}_{i}(k) \leq \xi^{*} \\ Q & \widetilde{r}_{i}(k)>\xi^{*}\end{cases}
$$

Under the condition that the parameters of the molecular MIMO communication system are determined, the whole detection process can be divided into three steps, i.e., calculating the elements in channel matrix $\mathbf{H}$ by (1), obtaining $\widetilde{\mathbf{R}}$ by premultiplying $\mathbf{R}$ with the pseudo inverse of channel matrix, and making a decision for each element $\widetilde{r}_{i}(k)$.

Besides, we are interested in the selection of the observation time $t_{s}$. In Section II, we assume an identical observation time for each reception antenna, i.e, the observation time for different CIRs in matrix $\mathbf{H}$ in (6) is $t_{s}$. Since all the received signals are used to demodulate the transmitted information, we expect each CIR in matrix $\mathbf{H}$ to be as large as possible. However, this cannot be achieved by an identical observation time. Considering that the observation time for CIRs of the same reception antenna should be the same, i.e., only one observation time can be exploited for one certain reception antenna, we choose the peak time which satisfies the maximum value of the superposition of CIRs at each reception antenna. Fig. 3 shows the CIRs with distance $d=\{8,10,12\} \mu \mathrm{m}$ 
and the superposition of them. It can be observed that for smaller initial distance $d$, the peak time is smaller but the peak concentration is larger. However, the superposition of the CIRs has only one peak, which can be chosen to obtain a higher signal to noise ratio (SNR) against the noise defined in (3).

Assuming there are $z$ CIRs with different distances $d_{1}, d_{2}, \ldots, d_{z}$, respectively, then the superposition of the CIRs has the following expression

$$
C I R_{\text {sum }}=\sum_{i=1}^{z} \frac{1}{(4 \pi D t)^{\frac{3}{2}}} \exp \left(-\frac{d_{i}^{2}}{4 D t}\right) .
$$

To find the extreme point of $C I R_{\text {sum }}$, we take derivative of (30) with respect to $t$ as

$$
\frac{\mathrm{d}}{\mathrm{d} t} C I R_{\text {sum }}=\sum_{i=1}^{z} \frac{\exp \left(-\frac{d_{i}^{2}}{4 D t}\right)}{(4 \pi D)^{\frac{3}{2}} t^{\frac{5}{2}}}\left(-\frac{3}{2}+\frac{d_{i}^{2}}{4 D t}\right) .
$$

The peak time of the superposition of the CIRs can be obtained as

$$
t_{\text {peak }}=\underset{t}{\arg \min }\left|\sum_{i=1}^{z} \frac{\exp \left(-\frac{d_{i}^{2}}{4 D t}\right)}{(4 \pi D)^{\frac{3}{2}} t^{\frac{5}{2}}}\left(-\frac{3}{2}+\frac{d_{i}^{2}}{4 D t}\right)\right| .
$$

The Newton-Raphson method [38] is considered to be capable to obtain accurate approximation for the root of a real-valued function and can be applied to obtain $t_{\text {peak }}$ in (32) numerically.

It should be noted that $t_{\text {peak }}$ is determined once the topology is determined but has different values at different reception antennas. Both the fixed observation time $t_{s}$ and the adaptive observation time $t_{\text {peak }}$ in (32) are utilized to analyze the error performance.

\section{ERROR PERFORMANCE WITHOUT ISI}

In this section, the error probability of the $\mathrm{ZF}$ detection approach is derived. In addition, the error performance of the proposed approach is compared with the detection scheme with transmission pairs in [11].

\section{A. Error Performance of ZF Detection}

For the detection with Gaussian noise, the error probability can be expressed as

$$
\begin{aligned}
P_{e} & =p_{1} P(0 \mid 1)+\left(1-p_{1}\right) P(1 \mid 0) \\
& =p_{1} \int_{-\infty}^{\xi^{*}} \frac{1}{\sqrt{2 \pi} \sigma} \exp \left(-\frac{\left(x-\mu_{1}\right)^{2}}{2 \sigma^{2}}\right) d x \\
& +\left(1-p_{1}\right) \int_{\xi^{*}}^{+\infty} \frac{1}{\sqrt{2 \pi} \sigma} \exp \left(-\frac{\left(x-\mu_{0}\right)^{2}}{2 \sigma^{2}}\right) d x \\
& =p_{1} Q\left(\frac{\mu_{1}-\xi^{*}}{\sigma}\right)+\left(1-p_{1}\right) Q\left(\frac{\xi^{*}-\mu_{0}}{\sigma}\right),
\end{aligned}
$$

where $p_{1}$ is the probability of transmitting symbol " 1 ", $\sigma$ is the standard deviation of the Gaussian noise and $Q(\cdot)$ denotes the $Q$-function.

By substituting $\sigma$ in (33) by the square root of $D\left(\widetilde{n}_{i}(k)\right)$ in (20), the error probability for the $k$ th symbol at the $i$ th transmission antenna, denoted by $P_{e i}^{k}$, can be obtained. The average error probability $\bar{P}_{e}$ of the whole MIMO system can be obtain by taking average on all the transmitted symbols

$$
\bar{P}_{e}=\frac{1}{q M} \sum_{k=1}^{q} \sum_{i=1}^{M} P_{e i}^{k}
$$

\section{B. Error Performance of Detection with Transmission Pairs}

In [11] and [16], a molecular MIMO communication system where transmission antennas and reception antennas form into several transmission pairs, is investigated. In this case, the $j$ th reception antenna is used to demodulate the signal from the $j$ th transmission antenna and signals from the other transmission antenna act as the ILI. The received signal at the $j$ th antenna in $k$ th symbol interval can be written as [11]

$$
r_{j}(k)=s_{j}(k) h_{j j}\left(t_{s}\right)+\sum_{i=1, i \neq j}^{M} s_{i}(k) h_{i j}\left(t_{s}\right)+n_{j}(k),
$$

where $s_{j}(k)$ and $s_{i}(k)$ are $Q$ or 0 corresponding to the bit transmitted in the $k$ th symbol interval.

The mean when transmitting bit " 0 " or " 1 " can be obtained by substituting $s_{j}(k)$ by " 0 " or " $Q$ " and averaging the influences of ILI and noise, which have the following expressions

$$
\begin{gathered}
\mu_{0}^{\prime}=\mu_{n}+\sum_{i=1, i \neq j}^{M} p_{1} Q h_{i j}\left(t_{s}\right), \\
\mu_{1}^{\prime}=Q h_{j j}\left(t_{s}\right)+\mu_{0}^{\prime} .
\end{gathered}
$$

The corresponding variance is therefore

$$
\sigma_{P}^{2}=\sigma_{0}^{2}=\sigma_{1}^{2}=\sigma_{n}^{2}+\underbrace{\sum_{i=1, i \neq j}^{M}\left(p_{1}-p_{1}^{2}\right) Q^{2} h_{i j}^{2}\left(t_{s}\right)}_{\text {variance of ILI }} .
$$

Then error probability with transmission pairs can be expressed as

$$
P_{e j}^{\prime}=p_{1} Q\left(\frac{\mu_{1}^{\prime}-\xi_{P}}{\sigma_{P}}\right)+\left(1-p_{1}\right) Q\left(\frac{\xi_{P}-\mu_{0}^{\prime}}{\sigma_{P}}\right)
$$

where $\xi_{P}$ can be obtained by substituting $\mu_{0}$ and $\mu_{1}$ in (28) by $\mu_{0}^{\prime}$ and $\mu_{1}^{\prime}$, respectively.

Similarly, we take the average error probability $\bar{P}_{e}^{\prime}$ into consideration, which can be express as

$$
\bar{P}_{e}^{\prime}=\frac{1}{M} \sum_{j=1}^{M} P_{e j}^{\prime}
$$

Theorem 2: The error probability of detection with transmission pairs approaches a constant when $Q$ goes to infinity, which means better error performance cannot be achieved by only increasing the number of molecules when transmitting "1". 
Proof 2:

$$
\begin{aligned}
& \lim _{Q \rightarrow \infty} \bar{P}_{e}^{\prime} \\
& =\lim _{Q \rightarrow \infty} \frac{1}{M} \sum_{j=1}^{M} p_{1} Q\left(\frac{Q h_{j j}\left(t_{s}\right)}{2 \sigma_{P}}\right)+\left(1-p_{1}\right) Q\left(\frac{Q h_{j j}\left(t_{s}\right)}{2 \sigma_{P}}\right) \\
& =\lim _{Q \rightarrow \infty} \frac{1}{M} \sum_{j=1}^{M} Q\left(\frac{Q h_{j j}\left(t_{s}\right)}{2 \sqrt{\sigma_{n}^{2}+\sum_{i=1, i \neq j}^{M} p_{1}\left(1-p_{1}\right) Q^{2} h_{i j}^{2}\left(t_{s}\right)}}\right) \\
& \stackrel{(a)}{=} \lim _{Q \rightarrow \infty} \frac{1}{M} \sum_{j=1}^{M} Q\left(\frac{Q h_{j j}\left(t_{s}\right)}{2 \sqrt{\sum_{i=1, i \neq j}^{M} p_{1}\left(1-p_{1}\right) Q^{2} h_{i j}^{2}\left(t_{s}\right)}}\right) \\
& =\frac{1}{M} \sum_{j=1}^{M} Q\left(\frac{h_{j j}\left(t_{s}\right)}{2 \sqrt{\sum_{i=1, i \neq j}^{M} p_{1}\left(1-p_{1}\right) h_{i j}^{2}\left(t_{s}\right)}}\right) .
\end{aligned}
$$

where equality (a) is hold because $\sigma_{n}^{2}$ is negligible compared with $\sum_{i=1, i \neq j}^{M} p_{1}\left(1-p_{1}\right) Q^{2} h_{i j}^{2}\left(t_{s}\right), h_{j j}\left(t_{s}\right)$ as well as $h_{i j}\left(t_{s}\right)$ are constant when the channel parameters are determined and thus $\bar{P}_{e}^{\prime}$ approaches a constant when $Q$ goes to infinity.

\section{ERROR PERFORMANCE WITH ISI}

In this section, both ILI and ISI are taken into account to investigate the entire performance of the considered molecular MIMO communication system. As described in Section III, ZF detection can effectively eliminate ILI. Therefore in this section, we analyze both ISI and ILI based on ZF detection. Due to the uncertainty of the transmitted bit sequence, the ISI at each reception antenna at a certain observation time is characterized as a random variable. The mean and variance of ISI are derived and combined with ZF detection to analyze the error performance.

\section{A. Mean of ISI}

As mentioned in section II, the transmitted information is split into $M$ streams and then fed to the transmission antennas, which makes the bit sequence transmitted at each transmission antenna quite random. The number of molecules transmitted at the $i$ th transmission antenna in the $k$ th symbol slot obeys the binomial distribution which is

$$
s_{i}(k) \sim \mathcal{B}\left(Q, p_{1}\right),
$$

where $\mathcal{B}$ denotes the binomial distribution. Then the mean of the ISI component can be calculated by take an average over all previous symbols, which can be expressed as

$$
\begin{aligned}
E\left(I S I_{j}(z)\right) & =E\left(\sum_{k=1}^{z-1} \sum_{i=1}^{M} s_{i}(k) h_{i j}\left(t_{s}-k T\right)\right) \\
& =\sum_{k=1}^{z-1} \sum_{i=1}^{M} E\left(s_{i}(k)\right) h_{i j}\left(t_{s}-k T\right) \\
& =\sum_{k=1}^{z-1} \sum_{i=1}^{M} p_{1} Q h_{i j}\left(t_{s}-k T\right) .
\end{aligned}
$$

TABLE I

Default PARAMETERs

\begin{tabular}{|c|c||c|c|}
\hline Parameter & Value & Parameter & Value \\
\hline \hline$D$ & $50 \mu \mathrm{m}^{2} / \mathrm{s}$ & $t_{s}$ & $0.5 \mathrm{~s}$ \\
\hline$d$ & $8 \mu \mathrm{m}$ & $\sigma_{n}^{2}$ & 100 \\
\hline$h$ & $6 \mu \mathrm{m}$ & $r$ & $2 \mu \mathrm{m}$ \\
\hline$p_{1}$ & 0.5 & $T$ & $1 \mathrm{~s}$ \\
\hline
\end{tabular}

\section{B. Variance of ISI}

For the considered molecular MIMO communication system, the ISI is a stochastic variable and brings uncertainty to the received signal. In other words, it introduces additional variance to the detection process. It should be noted that $s_{i}(k)$ for different $i$ and $k$ are independent because the transmitted bits are independent. Thus, the total variance of the ISI is the superposition of the ISI caused by each previous symbol and can be expressed as

$$
\begin{aligned}
D\left(I S I_{j}(z)\right) & =D\left(\sum_{k=1}^{z-1} \sum_{i=1}^{M} s_{i}(k) h_{i j}\left(t_{s}-k T\right)\right) \\
& =\sum_{k=1}^{z-1} \sum_{i=1}^{M} D\left(s_{i}(k)\right) h_{i j}^{2}\left(t_{s}-k T\right) \\
& =\sum_{k=1}^{z-1} \sum_{i=1}^{M} p_{1}\left(1-p_{1}\right) Q^{2} h_{i j}^{2}\left(t_{s}-k T\right) .
\end{aligned}
$$

In addition, the ISI component is directly related to signal transmitted in the previous $z-1$ time slots, but does not have relationship with the signal transmitted in the current time slot, which ensures independence between them. So the total variance for the detection is the summation of the variance of the signal in the current symbol duration and the variance of ISI.

For both detection schemes, ISI undergoes the same process as the external noise. Thus, the variance of the ISI can be combined with the variance caused by the noise and seen as an effective noise with an effective variance

$$
\sigma_{e f f}^{2}=\sigma_{n}^{2}+\sigma_{I S I}^{2},
$$

where $\sigma_{\text {eff }}^{2}$ and $\sigma_{I S I}^{2}$ are the effective variance and variance of ISI, respectively.

By substituting the effective noise variance $\sigma_{e f f}^{2}$ for the noise variance $\sigma_{n}^{2}$ and setting new threshold considering the mean of ISI, the error probability with ISI can be obtained.

\section{NUMERICAL RESULTS}

In this section, numerical results are presented to analyze the error performance of the proposed ZF detection. Error performances with or without ISI are investigated, respectively. For simplicity, we perform the numerical analysis on a $2 \times 4$ MIMO system or $2 \times 2$ MIMO system (reception antenna 2 and 3 are utilized), where the error performances of transmission antenna 1 and 2 are the same due to their symmetrical locations with respected to the receiver, as shown in Fig. 4. In this case, the average error probability of the whole system is the same as the error probability of either antenna. For all the 


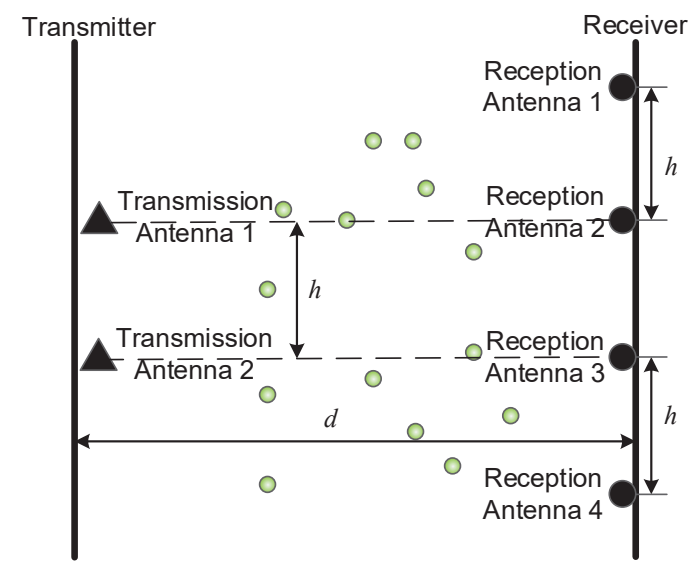

Fig. 4. The $2 \times 4$ molecular MIMO system in our numerical analysis.

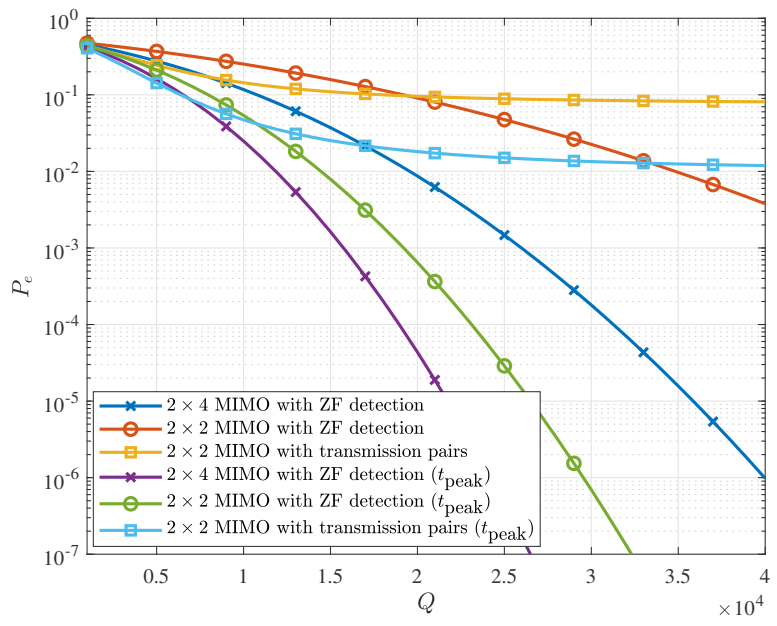

Fig. 5. Error performance of the detection approaches. The reception antennas in the $2 \times 2$ MIMO system are reception antenna 2 and 3 in Fig. 4 .

numerical results, we choose the default parameters in Table I, unless mentioned otherwise. Most parameters come from [17], such as the diffusion coefficient $D$, noise variance $\sigma_{n}^{2}$, radius of reception antenna $r$, probability of sending bit " 1 ", $p_{1}$ and symbol duration T. $d$ and $h$ are also on the same order of magnitude as [17].

\section{A. Error Performance without ISI}

In this subsection, the error performance of both detection schemes without ISI is analyzed. Specially, the lower bound of detection scheme with transmission pairs is introduced. $t_{s}$ and $t_{\text {peak }}$ are chosen as the observation time for both detection schemes to make a comparison. For detection with transmission pairs, $t_{\text {peak }}$ is chosen as the peak time of the CIR between transmission pairs, since we are expected to get the strongest signal strength without consideration of the ILI. In Fig. 5, the error probabilities of the ZF detection and the detection with transmission pairs are compared. The $2 \times 2$ MIMO system is a subsystem of $2 \times 4$ MIMO system where channel matrix $\mathbf{H}$ becomes $2 \times 2$ matrix. We observe that for all the curves, error probability decreases with the increase

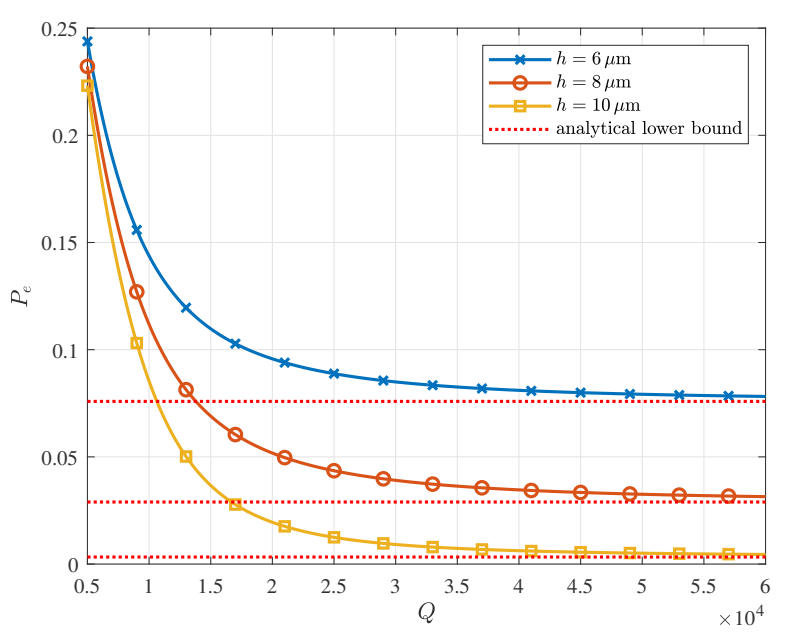

(a) Performance with identical observation time $t_{s}$.

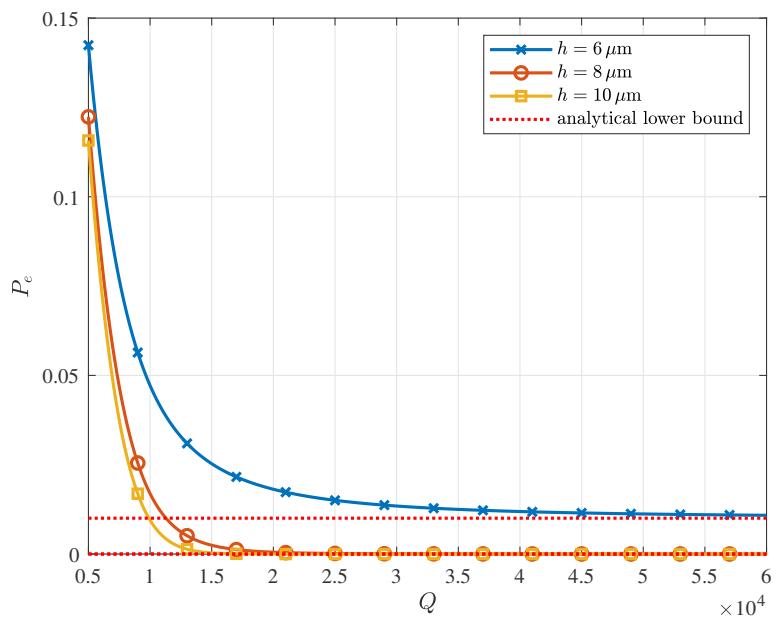

(b) Performance with adaptive observation time $t_{\text {peak }}$.

Fig. 6. Error performance and analytical lower bounds of detections with transmission pairs.

of $Q$. This is because larger $Q$ means larger signal strength as well as larger SNR. We also notice that for ZF detection, $2 \times 4$ MIMO system performs better than $2 \times 2 \mathrm{MIMO}$ system. This is because more reception antennas are used and more information can be utilized to make a more accurate decision. Moreover, for $2 \times 2$ MIMO system, the proposed $\mathrm{ZF}$ detection approach has better performance than the detection with transmission pairs when $Q$ is larger than $2 \times 10^{4}$. This is due to the fact that the increase of $Q$ leads to the increase of variance of ILI in (38) for detection with transmission pairs, but has no influence on the variance of the noise for ZF detection in (20). We also notice that the choice of $t_{\text {peak }}$ for different reception antennas leads to a better performance compared with an identical observation time $t_{s}$. This is due to the fact that signal strength is the strongest at all reception antennas, by choosing an adaptive observation time instead of an identical observation time. Stronger signal against the same noise results in a larger SNR and finally brings a better error performance. Considering an identical observation time, the 




Fig. 7. Error performance of ZF detection.

error probabilities for the detection scheme with transmission pairs with $h=\{6,8,10\} \mu \mathrm{m}$ is shown in Fig. 6(a). It validates Theorem 2 and shows there is a lower bound of the error probability when $Q$ becomes larger. We also notice that the lower bound is larger with smaller $h$. This is due to the fact that the ILI becomes larger when $h$ decreases, and therefore the variance caused by the ILI increases. Fig. 6(b) shows the error performance with adaptive observation time $t_{\text {peak }}$. A similar property can be observed compared with Fig. 6(a) but a smaller lower bound can be achieved for all the three cases. This is because the larger signal strength brought by adaptive observation time facilitates the detection with the same noise variance.

Fig. 7 shows the error probability of the ZF detection for $2 \times 4$ MIMO system with $d=\{6,8,10\} \mu \mathrm{m}$. It can be observed that better performance can be obtained with smaller $d$. This is because the CIR becomes larger when $d$ decreases, which results in the decrease of $h_{i j}^{\prime 2}$ in (20) and therefore the decrease of the variance. Smaller variance makes it easier to distinguish " 0 " and " $Q$ " and thus makes the decision more accurate. It can also be seen that the error probability decreases with the increase of $Q$ with no lower bound compared with the detection with transmission pairs in Fig. 6. This is because the variance of the noise in (20) is constant when the elements in $\mathbf{H}$ is determined and has no relationship with $Q$. As a result, the error probability decreases continuously with the increase of $Q$. Besides, the results show the error probability is almost the same for large $d$ (e.g., $10 \mu \mathrm{m}$ here), i.e., error performance cannot be enhanced significantly in this case. Therefore, for molecular MIMO communication systems with large horizontal distance $d$, there is no need to design the adaptive observation time for each reception antenna. For small $d$, we can make a tradeoff between the error performance and the complexity taken by employing the adaptive observation time.

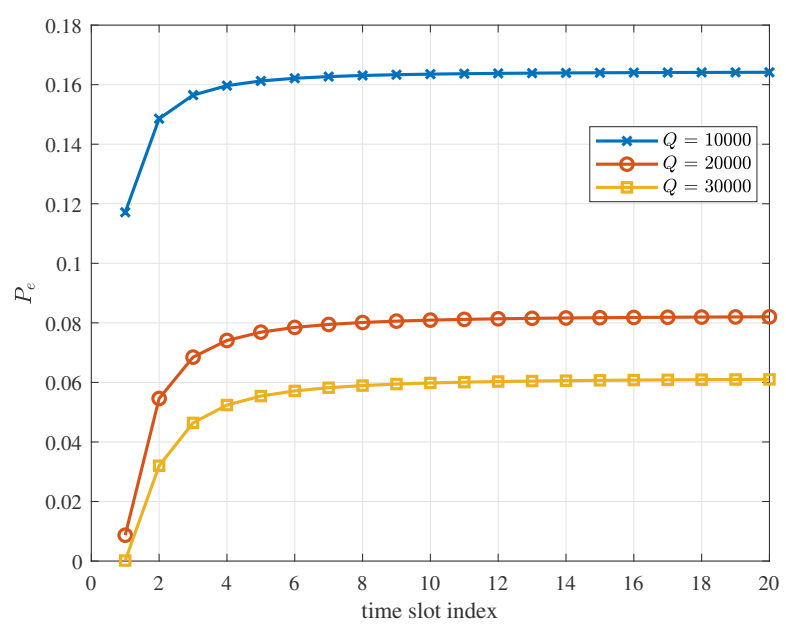

Fig. 8. Error performance of ZF detection with ISI in different time slot.

\section{B. Error Performance with ISI}

In this subsection, by regarding ISI as an effective noise, the error performance with ISI is investigated.

In Fig. 8, the error performance of ZF detection with ISI is shown with $Q=\{10000,20000,30000\}$. It can be observed that the error probability tends to be stable with the increase of time slot index, this is because the variance taken by ISI has minor changes when the time slot index becomes larger, since the influence of the first several symbols becomes negligible. We also notice that larger $Q$ brings better performance for the symbol in the same time slot. This is because larger $Q$ makes it easier to distinguish symbol " 0 " and " 1 " under the same interference.

The influence of symbol duration on the error performance is shown in Fig. 9. It can be seen that the error probability decreases with the increase of $T$. Moreover, the error probability tends to be stable when $T$ is lager (i.e., about $7 \mathrm{~s}$ here). This is due to the fact that ISI is smaller with larger symbol duration and can be neglected when the symbol duration becomes large enough, as been supposed in Section IV. We also notice that the error probability when $T$ is large enough is approximate to the error probability in the first time slot in Fig. 8. This is because, there is no ISI in the first symbol duration in Fig. 8 and ISI is extremely small for large $T$ in Fig. 9.

Compared with Fig. 7, the error probabilities in Fig. 8 and Fig. 9 seem much larger. This is because ISI is negligible by assuming large symbol duration $T$, or some other cases where ISI mitigation strategies are applied in Fig. 7. And the smallest error curve occurs with $d=6 \mu \mathrm{m}$ and observation time $t_{\text {peak }}$. But in Fig. 8 and Fig. 9, the identical observation time $t_{s}=$ $0.5 \mathrm{~s}$ and default $d=8 \mu \mathrm{m}$ are selected. The consistence in terms of error probability can be found when comparing the error probability curve (with $d=8 \mu \mathrm{m}$ and $t_{s}$ ) in Fig. 7 with the error probability in the first time slot in Fig. 8 and error probability when $T$ is large enough in Fig. 9, since ISI can be neglected in these two cases. And the same error probability which approximates to 0.12 can be found when $Q=10000$ for all the three figures. 


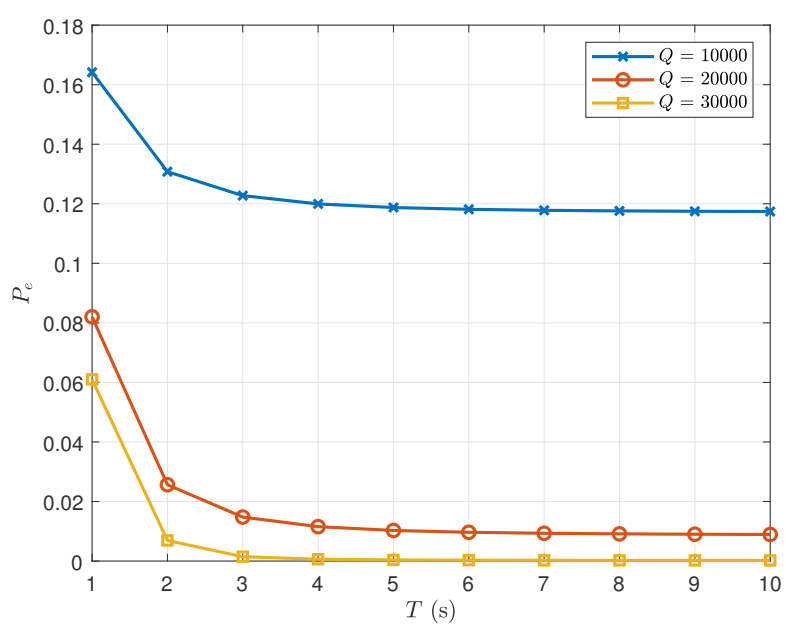

Fig. 9. Error performance of ZF detection with ISI for different symbol duration $T$.

\section{CONCLUSION}

Molecular MIMO communication is a significant topic in dealing with the low data rate in $\mathrm{MC}$ and an asymmetrical system topology makes the design more flexible. In this paper, we proposed the ZF detection approach for molecular MIMO communication system with asymmetrical topology. At the reception side, a fixed observation time as well as an adaptive observation time were exploited. Moreover, considering both ILI and ISI, the error probability of ZF detection was derived and was compared with the existing detection scheme where transmission pairs are assumed. Different from detection scheme with transmission pairs, error probability of ZF detection has no lower bound with the increase of $Q$. Numerical results show that better error performance can be obtained by ZF detection when the ILI is large.

\section{REFERENCES}

[1] T. Nakano, A. W. Eckford, and T. Haraguchi, Molecular communication. Cambridge, U.K.: Cambridge Univ. Press, 2013.

[2] I. F. Akyildiz, F. Brunetti, and C. Blázquez, "Nanonetworks: A new communication paradigm," Comput. Netw., vol. 52, no. 12, pp. 22602279, Aug. 2008.

[3] T. Nakano, M. J. Moore, F. Wei, A. V. Vasilakos, and J. Shuai, "Molecular communication and networking: Opportunities and challenges," IEEE Trans. Nanobiosci., vol. 11, no. 2, pp. 135-148, Jun. 2012.

[4] N. Farsad, H. B. Yilmaz, A. Eckford, C.-B. Chae, and W. Guo, "A comprehensive survey of recent advancements in molecular communication," IEEE Commun. Surveys Tuts., vol. 18, no. 3, pp. 1887-1919, Feb. 2016.

[5] Y. Chahibi, M. Pierobon, S. O. Song, and I. F. Akyildiz, "A molecular communication system model for particulate drug delivery systems," IEEE Trans. Biomed. Eng., vol. 60, no. 12, pp. 3468-3483, Jun. 2013.

[6] L. Lin, F. Huang, H. Yan, F. Liu, and W. Guo, "Ant-behavior inspired intelligent nanonet for targeted drug delivery in cancer therapy," IEEE Trans. NanoBiosci., 2020, DOI: 10.1109/TNB.2020.2984940.

[7] M. Hirabayashi, A. Nishikawa, F. Tanaka, M. Hagiya, H. Kojima, and K. Oiwa, "Design of molecular-based network robots-toward the environmental control," in Proc. 11th IEEE Int. Conf. Nanotechnol., Aug. 2011, pp. 313-318.

[8] B. Atakan, O. B. Akan, and S. Balasubramaniam, "Body area nanonetworks with molecular communications in nanomedicine," IEEE Commun. Mag., vol. 50, no. 1, pp. 28-34, Jan. 2012.
[9] T. Nakano, L. Lin, Y. Okaie, C. Wu, H. Yan, T. Hara, and K. Harumoto, "Random cell motion enhances the capacity of cell-cell communication," IEEE Trans. Mol., Biol. Multi-Scale Commun., vol. 5, no. 2, pp. 158162, Nov. 2019.

[10] G. Chang, L. Lin, and H. Yan, "Adaptive detection and ISI mitigation for mobile molecular communication," IEEE Trans. NanoBiosci., pp. 21-35, Jan. 2018.

[11] L. S. Meng, P. C. Yeh, K. C. Chen, and I. F. Akyildiz, "MIMO communications based on molecular diffusion," in Proc. IEEE Global Commun. Conf. (GLOBECOM), Dec. 2012, pp. 5380-5385.

[12] M. C. Gursoy, E. Basar, A. E. Pusane, and T. Tugcu, "Pulse positionbased spatial modulation for molecular communications," IEEE Commun. Lett., vol. 23, no. 4, pp. 596-599, Apr. 2019.

[13] Y. Huang, M. Wen, L.-L. Yang, C.-B. Chae, and F. Ji, "Spatial modulation for molecular communication," IEEE Trans. NanoBiosci., vol. 18, no. 3, pp. 381-395, Jul. 2019.

[14] M. C. Gursoy, E. Basar, A. E. Pusane, and T. Tugcu, "Index modulation for molecular communication via diffusion systems," IEEE Trans. Commun., vol. 67, no. 5, pp. 3337-3350, May 2019.

[15] S. M. Rouzegar and U. Spagnolini, "Channel estimation for diffusive MIMO molecular communications," in Proc. IEEE Eur. Conf. Netw. Commun. (EuCNC), Jun. 2017, pp. 1-5.

[16] B.-H. Koo, H. B. Yilmaz, C.-B. Chae, and A. Eckford, "Detection algorithms for molecular MIMO," in Proc. IEEE Int. Conf. Commun. (ICC), Jun. 2015, pp. 1122-1127.

[17] B.-H. Koo, C. Lee, H. B. Yilmaz, N. Farsad, A. Eckford, and C.-B. Chae, "Molecular MIMO: From theory to prototype," IEEE J. Sel. Areas Commun., vol. 34, no. 3, pp. 600-614, Mar. 2016.

[18] M. Damrath, H. B. Yilmaz, C.-B. Chae, and P. A. Hoeher, "Array gain analysis in molecular MIMO communications," IEEE Access, vol. 6, pp. 61 091-61 102, Oct. 2018.

[19] M. A. Mangoud, M. Lestas, and T. Saeed, "Molecular motors MIMO communications for nanonetworks applications," in Proc. IEEE Wireless Commun. Netw. Conf. (WCNC), Apr. 2018, pp. 1-5.

[20] C. Lee, H. B. Yilmaz, C.-B. Chae, N. Farsad, and A. Goldsmith, "Machine learning based channel modeling for molecular MIMO communications," in Proc. IEEE 18th Int. Workshop Signal Process. Adv. Wireless Commun. (SPAWC), Jul. 2017, pp. 1-5.

[21] H. B. Yilmaz, C. Lee, Y. J. Cho, and C.-B. Chae, "A machine learning approach to model the received signal in molecular communications," in Proc. IEEE Int. Black Sea Conf. Commun. Netw. (BlackSeaCom), Jun. 2017, pp. 1-5.

[22] X. Qian and M. Di Renzo, "Receiver design in molecular communications: An approach based on artificial neural networks," in Proc. 15th Int. Symp. Wireless Commun. Syst. (ISWCS), Aug. 2018, pp. 1-5.

[23] L. Lin, C. Yang, M. Ma, S. Ma, and H. Yan, "A clock synchronization method for molecular nanomachines in bionanosensor networks," IEEE Sensors J., vol. 16, no. 19, pp. 7194-7203, Oct. 2016.

[24] Z. Luo, L. Lin, W. Guo, S. Wang, F. Liu, and H. Yan, "One symbol blind synchronization in SIMO molecular communication systems," IEEE Wireless Commun. Lett., vol. 7, no. 4, pp. 530-533, Aug. 2018.

[25] D. C. Tosteson, Concepts and Models. Springer Science \& Business Media, 2012, vol. 1.

[26] M. Abbaszadeh, W. Li, L. Lin, I. White, P. Denissenko, P. J. Thomas, and W. Guo, "Mutual information and noise distributions of molecular signals using laser induced fluorescence," in Proc. IEEE Global Commun. Conf. (GLOBECOM), Dec. 2019, pp. 1-6.

[27] A. Noel, K. C. Cheung, and R. Schober, "Optimal receiver design for diffusive molecular communication with flow and additive noise," IEEE Trans. Nanobiosci., vol. 13, no. 3, pp. 350-362, Sept. 2014.

[28] — "Improving receiver performance of diffusive molecular communication with enzymes," IEEE Trans. Nanobiosci., vol. 13, no. 1, pp. 31-43, Mar. 2014.

[29] S. Huang, L. Lin, H. Yan, J. Xu, and F. Liu, "Statistical analysis of received signal and error performance for mobile molecular communication," IEEE Trans. Nanobiosci., vol. 18, no. 3, pp. 415-427, Jul. 2019.

[30] A. Noel, Y. Deng, D. Makrakis, and A. Hafid, "Active versus passive: Receiver model transforms for diffusive molecular communication," in Proc. IEEE Global Commun. Conf. (GLOBECOM), Dec. 2016, pp. 1-6.

[31] S. M. R. Rouzegar and U. Spagnolini, "Diffusive MIMO molecular communications: Channel estimation, equalization and detection," IEEE Trans. Commun., vol. 67, no. 7, pp. 4872-4884, Jul. 2019.

[32] D. Kilinc and O. B. Akan, "Receiver design for molecular communication," IEEE J. Sel. Areas Commun., vol. 31, no. 12, pp. 705-714, Dec. 2013. 
[33] R. Penrose, "A generalized inverse for matrices," in Mathematical proceedings of the Cambridge philosophical society, vol. 51, no. 3 . Cambridge Univ. Press, 1955, pp. 406-413.

[34] B. Noble, J. W. Daniel et al., Applied linear algebra. Prentice-Hall New Jersey, 1988, vol. 3.

[35] S. M. Alamouti, "A simple transmit diversity technique for wireless communications," IEEE J. Select. Areas Commun., vol. 16, pp. 14511458, Oct. 1998.

[36] B. Hassibi and B. M. Hochwald, "High-rate codes that are linear in space and time," IEEE Trans. Inf. Theory, vol. 48, no. 7, pp. 1804-1824, Jul. 2002.

[37] L. Lin, Q. Wu, F. Liu, and H. Yan, "Mutual information and maximum achievable rate for mobile molecular communication systems," IEEE Trans. Nanobiosci., vol. 17, no. 4, pp. 507-517, Oct. 2018.

[38] A. Gil, J. Segura, and N. M. Temme, Numerical methods for special functions. Siam, 2007, vol. 99. 
2020-06-22

\title{
Signal detection for molecular MIMO
} communications with asymmetrical topology

\author{
Wu, Cong
}

IEEE

Wu C, Lin L, Guo W, Yan H. (2020) Signal detection for molecular MIMO communications with asymmetrical topology. IEEE Transactions on Molecular, Biological, and Multi-Scale Communications, Volume 6, Issue 1, 2020, pp. 60-70

https://doi.org/10.1109/TMBMC.2020.3004030

Downloaded from Cranfield Library Services E-Repository 